ULM-TP/00-7

December 2000

\title{
Quantum cat maps with spin 1/2
}

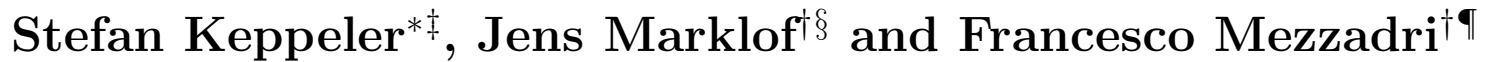

\author{
* Abteilung Theoretische Physik, Universität Ulm, Albert-Einstein-Allee 11, D-89069 Ulm, \\ Germany \\ † School of Mathematics, University Walk, University of Bristol, Bristol BS8 1TW, UK
}

\begin{abstract}
We derive a semiclassical trace formula for quantized chaotic transformations of the torus coupled to a two-spinor precessing in a magnetic field. The trace formula is applied to semiclassical correlation densities of the quantum map, which, according to the conjecture of Bohigas, Giannoni and Schmit, are expected to converge to those of the circular symplectic ensemble (CSE) of random matrices. In particular, we show that the diagonal approximation of the spectral form factor for small arguments agrees with the CSE prediction. The results are confirmed by numerical investigations.
\end{abstract}

PACS numbers: 05.45.Mt, 03.65.Sq

MSC numbers: 81Q50, 81Q20

\footnotetext{
${ }^{\ddagger}$ E-mail address: kep@physik.uni-ulm.de

${ }^{\S}$ E-mail address: j.marklof@bristol.ac.uk

IE-mail address: f.mezzadri@bristol.ac.uk
} 


\section{Introduction}

Bohigas, Giannoni and Schmit [1] have conjectured that the energy levels of quantized chaotic Hamiltonian systems are distributed like eigenvalues of random matrices. The choice of the correct matrix ensemble depends on the behaviour of the classical dynamics under time-reversal: the unitary ensemble (CUE) for systems without time-reversal invariance, the orthogonal or symplectic ensemble for systems with time-reversal invariance with integer or half-integer spin, respectively. Although there is much numerical evidence to support the conjecture for systems following CUE and COE statistics, relatively few studies are concerned with systems with half-integer spin [2, 3, 4, 5].

We shall here propose a simple model - a quantized map of the torus coupled to a spin precessing in a magnetic field - which is easily accessible both from the numerical and the analytical point of view: Suppose our particle's initial position is $q_{0}$ with momentum $p_{0}$. With $\left(p_{0}, q_{0}\right)$ fixed, we allow the spin to precess for one time unit in the magnetic field $\boldsymbol{B}\left(q_{0}\right)$; we then apply the Anosov map

$$
\phi: \mathbb{T}^{2} \rightarrow \mathbb{T}^{2}, \quad\left(\begin{array}{c}
p_{0} \\
q_{0}
\end{array}\right) \mapsto\left(\begin{array}{l}
p_{1} \\
q_{1}
\end{array}\right) \quad \bmod 1,
$$

keeping the spin fixed; thereafter the spin is allowed to precess again for one time unit in $\boldsymbol{B}\left(q_{1}\right)$, and so on.

The idea here is that, due to the chaotic dynamics of $\phi$, the values of the magnetic field at the iterated positions $q_{0}, q_{1}, q_{2}, \ldots$ are essentially uncorrelated and the spin precession therefore behaves like a random walk in $\mathrm{SU}(2)$. The ergodic properties of such constructions, so-called skew products, are well understood [6, 7, 8, 9].

The quantization of our model in terms of unitary $2 N \times 2 N$ matrices $\mathrm{U}$ is straightforward (section 2). In section 3 we discuss in detail those anti-unitary operators $\mathrm{T}$ which correspond to time-reversal symmetries in that $\mathrm{T}^{-1} \mathrm{UT}=\mathrm{U}^{-1}$. If $\mathrm{T}$ is the only such symmetry present, and if in addition $\mathrm{T}^{2}=-\mathbb{1}_{2 N}$, one expects the spacing distribution for the eigenvalues of $\mathrm{U}$ to converge in the semiclassical limit $N \rightarrow \infty$ to that of the symplectic ensemble CSE.

Indeed, for generic Anosov maps $\phi$ we numerically observe a transition from COE to

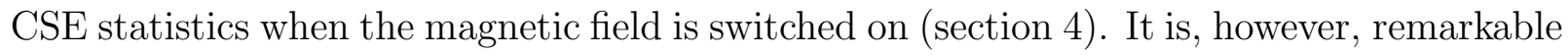
that even unperturbed cat maps, whose spectrum is highly degenerate [10, 11, exhibit CSE statistics for non-zero magnetic fields.

The remainder of our paper is devoted to the semiclassical analysis of spectral correlation densities. In section 5 we derive a semiclassical trace formula which expresses sums over eigenvalues of $U$ in terms of sums over fixed points of the classical Anosov map $\phi$. We observe that the fixed points remain unchanged when switching on the magnetic field but that additional weight factors, taking care of the spin contribution, appear. In the case of flows an analogous observation has been made in 12, 13. In section 6 the trace formula is employed to investigate two-point correlations of the eigenvalues of $U$, with focus on the behaviour of the spectral form factor for small arguments. In particular, we show that the diagonal approximation [14, 15] reproduces the random matrix prediction if the magnetic 
field is sufficiently generic. We essentially follow the line of arguments in [16], where the form factor for continuous-time dynamics is discussed. However, the assumption in [16] that the skew product dynamics must be mixing is replaced by a simpler condition. In the final section 7 we discuss the interesting fact that the diagonal approximation also works if the spin precession does not become equidistributed in $\mathrm{SU}(2)$, but only takes a finite number of distinct values in $\mathrm{SU}(2)$.

\section{Maps with spin}

The Hamiltonian of a particle with spin $\frac{1}{2}$ in a magnetic field $\boldsymbol{B}(q)$ is given by (in units where $2 m c / e=1$ )

$$
\mathrm{H}=\mathrm{H}_{0} \mathbb{1}_{2}-\hbar \boldsymbol{\sigma} \cdot \boldsymbol{B}(q),
$$

where $\mathrm{H}_{0}$ is a scalar Hamiltonian, and the second term describes the interaction between the magnetic moment of spin and the magnetic field $\boldsymbol{B}(q)$. Here $\mathbb{1}_{2}$ denotes the $2 \times 2$ unit matrix, and $\boldsymbol{\sigma}$ is the vector of Pauli matrices

$$
\sigma_{x}=\left(\begin{array}{ll}
0 & 1 \\
1 & 0
\end{array}\right), \quad \sigma_{y}=\left(\begin{array}{cc}
0 & -\mathrm{i} \\
\mathrm{i} & 0
\end{array}\right), \quad \sigma_{z}=\left(\begin{array}{cc}
1 & 0 \\
0 & -1
\end{array}\right) .
$$

We are interested in modeling free spin precession interrupted only by periodic deltashaped kicks at integer times $t=n \in \mathbb{Z}$. Such a system is represented by the time dependent Hamiltonian

$$
\mathrm{H}(t)=\sum_{n \in \mathbb{Z}} \mathrm{H}_{0} \mathbb{1}_{2} \delta(t-n)-\hbar \boldsymbol{\sigma} \cdot \boldsymbol{B}(q),
$$

cf. [4, 5]. The Floquet operator which propagates the system, e.g., from time $t=-\frac{1}{2}$ to $t=\frac{1}{2}$, is then given by

$$
\mathrm{U}=\mathrm{U}_{\mathrm{s}} \mathrm{U}_{\mathrm{t}} \mathrm{U}_{\mathrm{s}}, \quad \text { with } \quad \mathrm{U}_{\mathrm{s}}=\mathrm{e}^{\frac{\mathrm{i}}{2} \boldsymbol{\sigma} \cdot \boldsymbol{B}(q)} \quad \text { and } \quad \mathrm{U}_{\mathrm{t}}=\mathrm{e}^{-\frac{\mathrm{i}}{\hbar} \mathrm{H}_{0}} \mathbb{1}_{2}
$$

In this paper, the translational dynamics $U_{t}$ will be represented by a quantized map of the torus $\mathbb{T}^{2}$, which is given by a unitary operator $\mathrm{U}_{N}$ acting on an $N$ dimensional Hilbert space $\mathcal{H}_{N} \simeq \mathbb{C}^{N}$. The dimension of the Hilbert space $\mathcal{H}_{N}$ and Planck's constant are related via the condition $2 \pi \hbar N=1$. (We refer the reader to appendix A for more details on the quantization of maps on the torus.) For instance, in the case of the map

$$
A:\left(\begin{array}{l}
p \\
q
\end{array}\right) \mapsto\left(\begin{array}{ll}
2 & 3 \\
1 & 2
\end{array}\right)\left(\begin{array}{l}
p \\
q
\end{array}\right)=\left(\begin{array}{c}
2 p+3 q \\
p+2 q
\end{array}\right) \quad \bmod 1
$$

the action of $U_{N}$ on functions $\Phi \in \mathcal{H}_{N}$ is given by 17]

$$
\left[\mathrm{U}_{N}(A) \Phi\right](Q)=\left(\frac{1}{\mathrm{i} N}\right)^{1 / 2} \sum_{Q^{\prime}=0}^{N-1} \exp \left[\frac{2 \pi \mathrm{i}}{N}\left(Q^{2}-Q Q^{\prime}+Q^{\prime 2}\right)\right] \Phi\left(Q^{\prime}\right)
$$


$Q=0, \ldots, N-1$. We shall also investigate the perturbed cat map

$$
\phi: \rho \circ A \circ \rho
$$

with perturbation

$$
\boldsymbol{\rho}:\left(\begin{array}{c}
p \\
q
\end{array}\right) \mapsto\left(\begin{array}{c}
p+\frac{k}{2} f(q) \\
q
\end{array}\right) \quad \bmod 1
$$

where $f$ is a bounded periodic function and $k$ measures the strength of perturbation. Since Anosov systems are structurally stable, $\phi$ remains Anosov if $k$ is small enough. The quantization of $\phi$ is represented by 18

$$
\mathrm{U}_{N}(\phi)=\mathrm{U}_{N}(\boldsymbol{\rho}) \mathrm{U}_{N}(A) \mathrm{U}_{N}(\boldsymbol{\rho})
$$

with

$$
\left[\mathrm{U}_{N}(\boldsymbol{\rho}) \Phi\right](Q)=\mathrm{e}^{\pi \mathrm{i} N k S\left(\frac{Q}{N}\right)} \Phi(Q)
$$

and $f(q)=\frac{\mathrm{d}}{\mathrm{d} q} S(q)$.

The propagators $\mathrm{U}_{\mathrm{t}}(A)$ and $\mathrm{U}_{\mathrm{t}}(\phi)$ now act on a two-spinor $\Psi \in \mathbb{C}^{2} \otimes \mathcal{H}_{N}$ simply by

$$
\mathrm{U}_{\mathrm{t}}(A) \Psi=\left(\begin{array}{c}
\mathrm{U}_{N}(A) \Phi_{1} \\
\mathrm{U}_{N}(A) \Phi_{2}
\end{array}\right), \quad \mathrm{U}_{\mathrm{t}}(\phi) \Psi=\left(\begin{array}{c}
\mathrm{U}_{N}(\phi) \Phi_{1} \\
\mathrm{U}_{N}(\phi) \Phi_{2}
\end{array}\right), \quad \Psi=\left(\begin{array}{c}
\Phi_{1} \\
\Phi_{2}
\end{array}\right), \quad \Phi_{1}, \Phi_{2} \in \mathcal{H}_{N}
$$

and the action of $\mathrm{U}_{\mathrm{s}}$ is straightforwardly given by

$$
\left[\mathrm{U}_{\mathrm{s}} \Psi\right](Q)=\mathrm{e}^{\frac{\mathrm{i}}{2} \sigma \cdot B\left(\frac{Q}{N}\right)} \Psi(Q) .
$$

\section{Anti-unitary symmetries}

Following Bohigas, Giannoni and Schmit [1], we expect that the eigenvalue statistics of the quantized map U converge to those of the circular random matrix ensembles, CUE, COE, or CSE. The choice of the correct ensemble depends on whether the quantum map possesses an anti-unitary symmetry T. In particular, the spectral fluctuations are expected to agree with those of the CSE ensemble if $\mathrm{T}^{2}=-\mathbb{1}_{2 N}$. In this section we discuss the necessary conditions to be imposed on the classical map $\boldsymbol{\phi}$ and on the magnetic field $\boldsymbol{B}(q)$ in order to observe CSE statistics.

To this end consider an Anosov map $\phi$ invariant under time reversal, i.e.

$$
\boldsymbol{\tau} \circ \boldsymbol{\phi} \circ \boldsymbol{\tau}=\boldsymbol{\phi}^{-1}, \quad \text { where } \quad \boldsymbol{\tau}:\left(\begin{array}{l}
p \\
q
\end{array}\right) \mapsto\left(\begin{array}{c}
-p \\
q
\end{array}\right) .
$$


Moreover, suppose that the map $\phi$ is also invariant under inversion, i.e.

$$
\boldsymbol{\pi} \circ \boldsymbol{\phi} \circ \boldsymbol{\pi}=\boldsymbol{\phi} \quad \text { with } \quad \boldsymbol{\pi}:\left(\begin{array}{c}
p \\
q
\end{array}\right) \mapsto\left(\begin{array}{l}
-p \\
-q
\end{array}\right) .
$$

Then, $\phi$ will also be invariant under a transformation with

$$
\tilde{\tau}=\pi \circ \boldsymbol{\tau}
$$

which we will call non-conventional time-reversal, see, e.g. [19] for a general discussion. For spinless motion the quantum time reversal operator $\mathrm{K}$ is simply complex conjugation,

$$
[K \Psi](Q)=\bar{\Psi}(Q),
$$

and the operator corresponding to $\tilde{\tau}$ is given by

$$
[\tilde{\mathrm{K}} \Psi](Q)=\bar{\Psi}(-Q) .
$$

Moreover, since $\boldsymbol{\tau}$ and $\tilde{\boldsymbol{\tau}}$ are symmetries of $\phi$, the quantum map $\mathrm{U}_{\mathrm{t}}(\phi)$ will be invariant under $\mathrm{K}$ and $\tilde{\mathrm{K}}$, i.e.

$$
\mathrm{KU}_{\mathrm{t}}(\phi) \mathrm{K}=\mathrm{U}_{\mathrm{t}}^{-1}(\phi), \quad \tilde{\mathrm{K}} \mathrm{U}_{\mathrm{t}}(\phi) \tilde{\mathrm{K}}=\mathrm{U}_{\mathrm{t}}^{-1}(\phi) .
$$

In general, however, the coupled map $\mathrm{U}(\phi)=\mathrm{U}_{\mathrm{s}} \mathrm{U}_{\mathrm{t}}(\phi) \mathrm{U}_{\mathrm{s}}$ is not invariant under these symmetries, because

$$
\begin{aligned}
{\left[\mathrm{K} \mathrm{U}_{\mathrm{s}} \mathrm{K} \Psi\right](Q) } & =\mathrm{e}^{-\frac{\mathrm{i}}{2} \boldsymbol{\sigma} \cdot \tilde{\boldsymbol{B}}\left(\frac{Q}{N}\right)} \Psi(Q), \\
{\left[\tilde{\mathrm{K}} \mathrm{U}_{\mathrm{s}} \tilde{\mathrm{K}} \Psi\right](Q) } & =\mathrm{e}^{-\frac{\mathrm{i}}{2} \boldsymbol{\sigma} \cdot \tilde{\boldsymbol{B}}\left(-\frac{Q}{N}\right)} \Psi(Q),
\end{aligned}
$$

where $\tilde{\boldsymbol{B}}(q)=\left(B_{x}(q),-B_{y}(q), B_{z}(q)\right)$. Since we are concerned with system with spin, we should also invert the direction of spin when reversing time. Let us consequently define the modified time-reversal operators $\mathrm{T}$ and $\tilde{\mathrm{T}}$ by

$$
[\mathrm{T} \Psi](Q)=\mathrm{e}^{\mathrm{i} \pi \sigma_{y} / 2} \bar{\Psi}(Q)=\mathrm{i} \sigma_{y} \bar{\Psi}(Q), \quad[\tilde{\mathrm{T}} \Psi](Q)=\mathrm{i} \sigma_{y} \bar{\Psi}(-Q) .
$$

Note that $\mathrm{T}^{2}=\tilde{\mathrm{T}}^{2}=-\mathbb{1}_{2 N}$, as needed for CSE statistics. For these operators one obtains

$$
\begin{aligned}
& {\left[\mathrm{T}^{-1} \mathrm{U}_{\mathrm{s}} \mathrm{T} \Psi\right](Q)=\mathrm{e}^{\frac{\mathrm{i}}{2} \boldsymbol{\sigma} \cdot \boldsymbol{B}\left(\frac{Q}{N}\right)} \Psi(Q),} \\
& {\left[\tilde{\mathrm{T}}^{-1} \mathrm{U}_{\mathrm{s}} \tilde{\mathrm{T}} \Psi\right](Q)=\mathrm{e}^{\frac{\mathrm{i}}{2} \boldsymbol{\sigma} \cdot \boldsymbol{B}\left(-\frac{Q}{N}\right)} \Psi(Q) .}
\end{aligned}
$$

From (3.9a) we easily see that $\mathrm{T}^{-1} \mathrm{U}_{\mathrm{s}} \mathrm{T} \neq \mathrm{U}_{\mathrm{s}}^{-1}$ for non-zero magnetic fields, since obviously $\boldsymbol{B}(q) \neq-\boldsymbol{B}(q)$. However, rel. (3.9b) implies that $\tilde{\mathrm{T}}^{-1} \mathrm{U}_{\mathrm{s}} \tilde{\mathrm{T}}=\mathrm{U}_{\mathrm{s}}^{-1}$ for odd magnetic field, i.e. if $\boldsymbol{B}(-q)=-\boldsymbol{B}(q)$. 
In addition we have to make sure that there is no further anti-unitary symmetry, in particular the system must neither be invariant under $\mathrm{K}$ nor under $\tilde{\mathrm{K}}$. If $B_{y}(q)$ vanishes identically then $\tilde{\boldsymbol{B}}(q)=\boldsymbol{B}(q)$ and by (3.7a) the spin part is also invariant under $\mathrm{K}$, which should be avoided. The last argument is of course independent of the choice of the coordinate system, and hence we require that there is no vector $\boldsymbol{r} \neq 0$ such that $\boldsymbol{r} \cdot \boldsymbol{B}(q)=0$ for all $q$, i.e. the magnetic field $\boldsymbol{B}(q)$ must have linearly independent components.

To summarize, in order to observe CSE spectral distributions we shall require that $\tilde{\boldsymbol{\tau}}$ is a symmetry of the classical map $\boldsymbol{\phi}$, that the magnetic field $\boldsymbol{B}(q)$ is odd and that its components are independent functions.

\section{Spectral statistics}

After having clarified under which conditions we expect to see CSE statistics we will now test our arguments in a numerical experiment for the quantized cat map $A$ and its perturbation $\phi$. A simple choice of the shear in (2.8) is, e.g., given by

$$
f(q)=\frac{1}{2 \pi} \sin 2 \pi q .
$$

The map $\phi$ will be Anosov if 18

$$
k<k_{\max }=\frac{\sqrt{3}-1}{\sqrt{5}}=0.327 \ldots
$$

The only symmetries of the cat map (2.5) are time-reversal $\boldsymbol{\tau}(3.1)$, inversion $\boldsymbol{\pi}(3.2)$, and non-conventional time-reversal $\tilde{\tau}$ (3.3). The choice of the perturbation (4.1), with $f$ being odd, conserves all symmetries $\boldsymbol{\tau}, \tilde{\boldsymbol{\tau}}$ and $\boldsymbol{\pi}$.

Now, in order to observe CSE statistics, the spin precession must be caused by a magnetic field which is odd and periodic in $q$ and whose components are independent functions (cf. section 3). These constraints guarantee that the only anti-unitary symmetry

of the quantum system is $\tilde{\mathrm{T}}(\overline{3.8})$. For our numerical calculation we chose the magnetic field

$$
\boldsymbol{B}(q)=\left(\begin{array}{c}
\sin (2 \pi q) \\
\sin (4 \pi q) \\
\sin (6 \pi q)
\end{array}\right)
$$

which clearly satisfies these requirements.

We now combine $\mathrm{U}_{N}(A)$ and $\mathrm{U}_{N}(\phi)$ with the spin precession propagator $\mathrm{U}_{\mathrm{s}}$ as in (2.4) to obtain the quantum maps

$$
\mathrm{U}(A)=\mathrm{U}_{\mathrm{s}} \mathrm{U}_{\mathrm{t}}(A) \mathrm{U}_{\mathrm{s}} \quad \text { and } \quad \mathrm{U}(\phi)=\mathrm{U}_{\mathrm{s}} \mathrm{U}_{\mathrm{t}}(\phi) \mathrm{U}_{\mathrm{s}},
$$

where

$$
\mathrm{U}_{\mathrm{t}}(A)=\left(\begin{array}{cc}
\mathrm{U}_{N}(A) & 0 \\
0 & \mathrm{U}_{N}(A)
\end{array}\right) \quad \text { and } \quad \mathrm{U}_{\mathrm{t}}(\phi)=\left(\begin{array}{cc}
\mathrm{U}_{N}(\phi) & 0 \\
0 & \mathrm{U}_{N}(\phi)
\end{array}\right)
$$


The matrix elements of $\mathrm{U}(A)$ and $\mathrm{U}(\boldsymbol{\phi})$ are easily worked out using (2.6) - (2.12). If $N$ is divisible by four, there exist simple relations between the matrix elements of the propagators in eqs. (2.6) and (2.9) 20] which lead to invariance with respect to the unitary operator

$$
[\mathrm{R} \Psi](Q)=\mathrm{ie}^{\mathrm{i} \pi Q} \Psi(Q+N / 2)
$$

This operator obeys the relations

$$
[\mathrm{U}(A), \mathrm{R}]=0, \quad[\mathrm{U}(\phi), \mathrm{R}]=0, \quad[\mathrm{R}, \tilde{\mathrm{T}}]=0 \quad \text { and } \quad \mathrm{R}^{2}=-\mathbb{1}_{2 N} .
$$

As a consequence in this case the statistics of the spectra of $\mathrm{U}(A)$ and $\mathrm{U}(\phi)$ would follow those of the CUE ensemble, cf. [19]. Thus, we shall require that $N \not \equiv 0 \bmod 4$.

Studying the spectral statistics of quantum maps means looking at the distributions of the eigenangles $\left\{\alpha_{1}, \ldots, \alpha_{2 N}\right\}$. In order to compare energy fluctuations in different systems, the spectra must be unfolded, that is the energy levels are rescaled so that the mean level spacing is one. (The spacing $s$ is defined as the distance between two consecutive levels.) Moreover, in order to observe universal distributions the spectra must be desymmetrized, that is only energy levels with the same quantum numbers corresponding to all mutually commuting unitary symmetries must be considered. Therefore, when analyzing the spectra of $\mathrm{U}_{N}(A)$ and $\mathrm{U}(\phi)$ in the absence of spin dynamics, we have to take into account only eigenphases which correspond to the same eingenvalue $P= \pm 1$ of $\boldsymbol{\pi}$. For systems with halfinteger spin and time-reversal invariance an additional proviso must be taken into account: each energy level has at least multiplicity two (Kramers' degeneracy [21]) because the square of time-reversal operators $\mathrm{T}$ for systems with half-integer spin being $-\mathbb{1}_{2 N}$ causes $T \Psi$ to be orthogonal to $\Psi$ 222. The statistical analysis, however, must be performed on a spectrum obtained by removing such degeneracy. Compare section 6 for details.

Let us briefly describe the spectral distributions of $\mathrm{U}_{N}(A)$ and $\mathrm{U}_{N}(\phi)$ in the absence of spin dynamics. The spectra of quantum cat maps $\mathrm{U}_{N}(A)$ are well known to be highly nongeneric, and in particular do not follow any universal distribution [23]. The reason for this untypical behaviour is the high number of quantum symmetries - about $O(N)$ many 10, 11 - which commute with the quantum propagator $\mathrm{U}_{N}(A)$. Such arithmetical symmetries can easily be broken by slightly perturbing the classical map [18, and the expected COE random matrix statistics are recovered. This behaviour is evident in fig. 1, where the consecutive level spacing distribution $p(s)$ and the integrated level spacing distribution

$$
I(s)=\int_{0}^{s} p\left(s^{\prime}\right) \mathrm{d} s^{\prime}
$$

are plotted for the quantum maps (2.6) and (2.9).

Fig. 2 shows the spectral statistics of $\mathrm{U}(A)$ and $\mathrm{U}(\phi)$ defined in (4.4) with the magnetic field given by (4.3). Clearly, in both cases the spectral distributions nicely follow CSE statistics, as predicted. It is remarkable that the non-generic behaviour of the quantum cat map without spin (figs. 1(a) and 1(b)) has disappeared, even though the spin coupling 


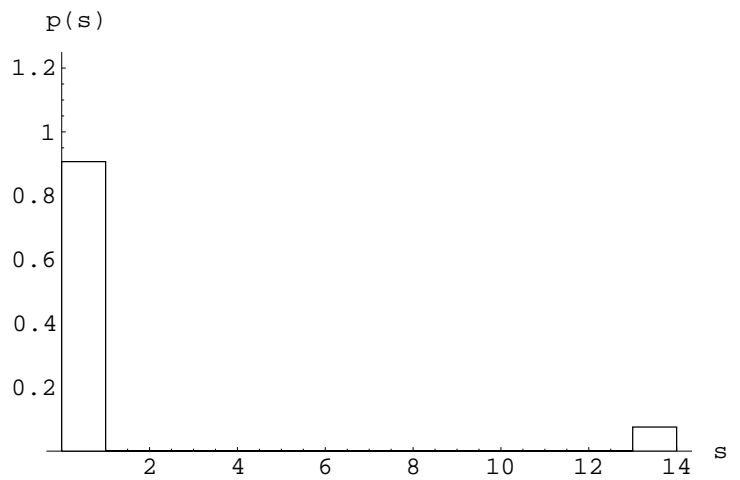

(a) Spacing distribution of the quantum cat map (2.6) with $P=+1$.

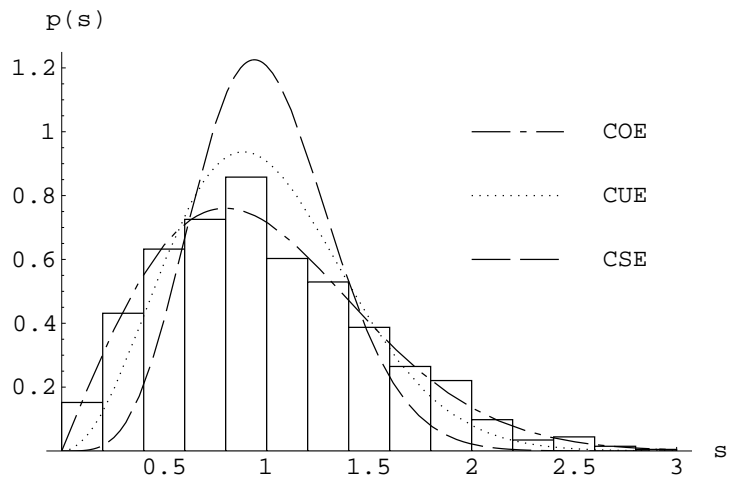

(c) Spacing distribution of the quantum map (2.9) with $\mathrm{k}=0.32$ and $P=+1$.

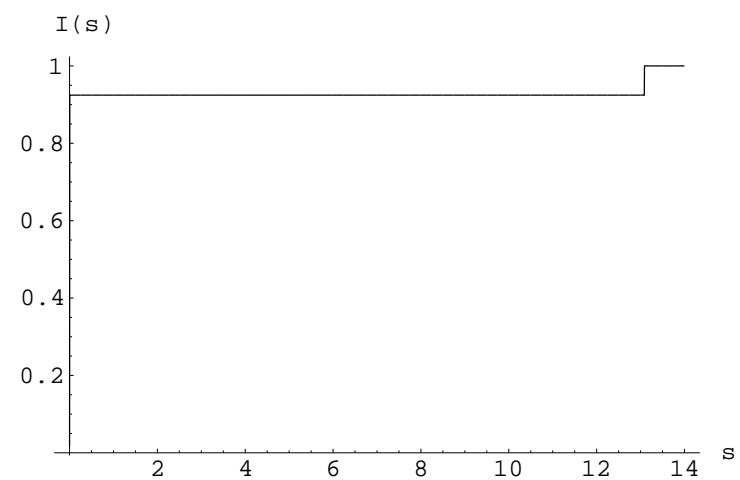

(b) Cumulative spacing distribution of the quantum cat map (2.6) with $P=+1$.

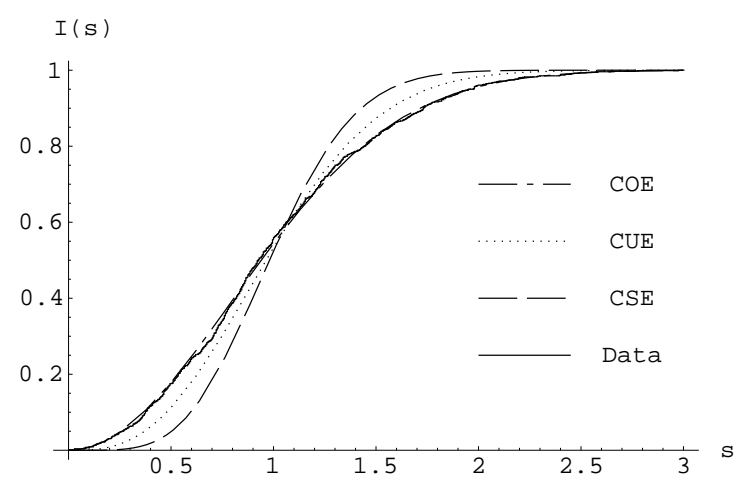

(d) Cumulative spacing distribution of the quantum map (2.9) with $\mathrm{k}=0.32$ and $P=+1$.

Figure 1: Local spectral statistics of the quantum cat map (2.6) (above) and of its perturbation (2.9) (below). The eigenangles belong to states with the same eigenvalue of $\boldsymbol{\pi}$ and their number is $N=1021$. 


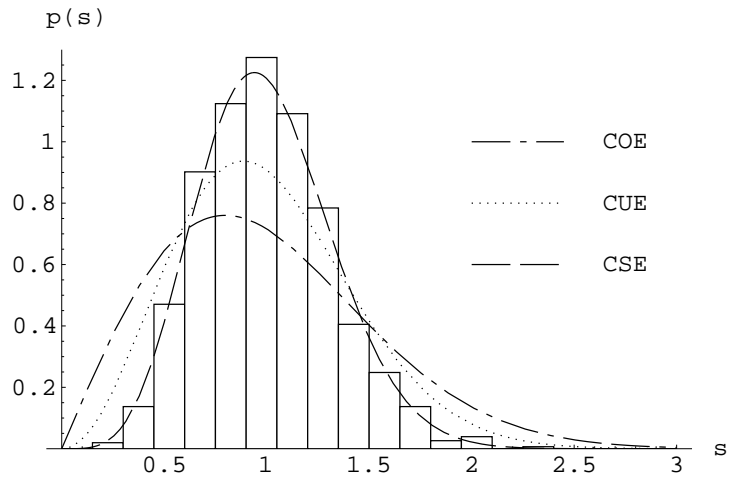

(a) Spacing distribution of the coupled map $\mathrm{U}(A)$.

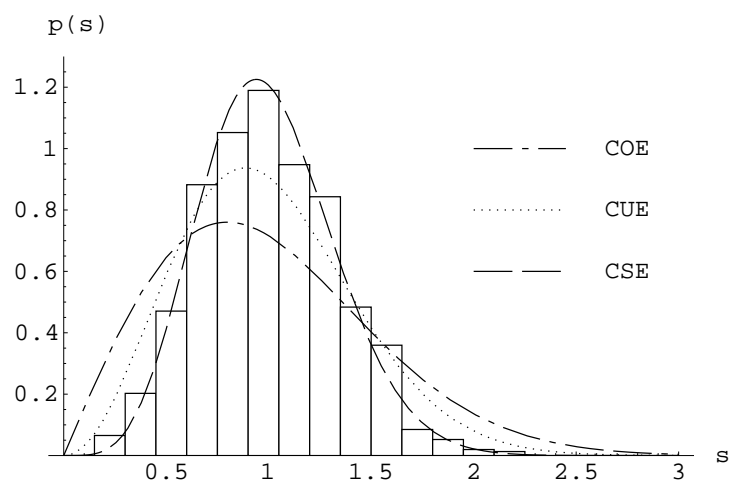

(c) Spacing distribution of the coupled map $\mathrm{U}(\phi)$ with $\mathrm{k}=0.32$.

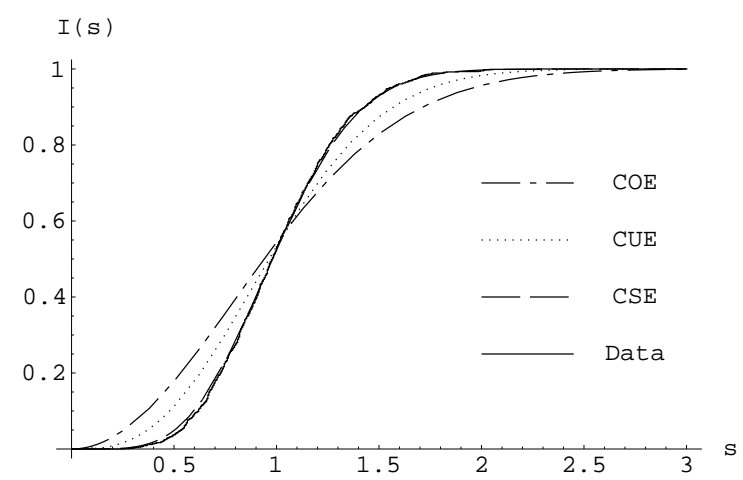

(b) Cumulative spacing distribution of the coupled map $\mathrm{U}(A)$.

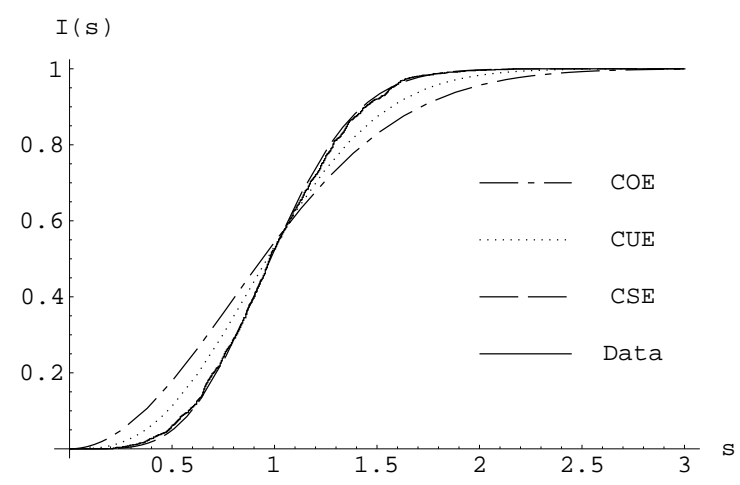

(d) Cumulative spacing distribution of the coupled map $\mathrm{U}(\phi)$ with $\mathrm{k}=0.32$.

Figure 2: Local spectral statistics of the quantum cat map (above) and of its perturbation (below) when the spin dynamics is introduced. The number of eigenangles $N$ is 1021 . 
is of sub-principal order in $\hbar$ and therefore does not affect the highly degenerate classical dynamics. The semiclassical arguments in the following sections will explain the CSE statistics for generic maps $\phi$, but in the case of the unperturbed cat map $A$ the situation is more subtle. We will briefly return to this point in section 0 when discussing piecewise constant magnetic fields.

\section{$5 \quad$ Semiclassical trace formula}

The purpose of this section is to derive a trace formula for the quantum maps (4.4). Since Berry's study of the spectral rigidity [15] based on Gutzwiller's trace formula [24], trace formulae are the main semiclassical tools in analyzing spectral correlations. In the case of flows trace formulae including spin contributions have been derived in [13 for the Pauli and the Dirac equation.

In what follows $\phi$ can be any Anosov map on $\mathbb{T}^{2}$. The magnetic field $\boldsymbol{B}$ will be left generic too, except that, for simplicity, it should be a function of $q$ only. Our goal is to determine $\operatorname{Tr}\left[\mathrm{U}^{n}(\phi)\right]$ as a sum over periodic orbits. For this purpose it is more convenient to consider the operator

$$
\tilde{\mathrm{U}}(\phi)=\mathrm{U}_{\mathrm{t}}(\phi) \mathrm{U}_{\mathrm{s}}^{2}
$$

Since $\tilde{U}(\phi)$ is unitarily conjugate to $\mathrm{U}(\boldsymbol{\phi})$, clearly $\operatorname{Tr}\left[\mathrm{U}^{n}(\phi)\right]=\operatorname{Tr}\left[\tilde{\mathrm{U}}^{n}(\phi)\right]$.

Let $\mathrm{Op}_{N}(f)$ denote the Weyl quantization of a classical observable $f \in C^{\infty}\left(\mathbb{T}^{2}\right.$ ) (see appendix A). For a $2 \times 2$ matrix

$$
g(\boldsymbol{z})=\left(\begin{array}{ll}
g_{11}(\boldsymbol{z}) & g_{12}(\boldsymbol{z}) \\
g_{21}(\boldsymbol{z}) & g_{22}(\boldsymbol{z})
\end{array}\right), \quad \boldsymbol{z}=(p, q) \in \mathbb{T}^{2},
$$

whose elements are smooth functions on $\mathbb{T}^{2}$, we then define

$$
\mathrm{Op}_{N}(g)=\left(\begin{array}{ll}
\mathrm{Op}_{N}\left(g_{11}\right) & \mathrm{Op}_{N}\left(g_{12}\right) \\
\mathrm{Op}_{N}\left(g_{21}\right) & \mathrm{Op}_{N}\left(g_{22}\right)
\end{array}\right)
$$

which semiclassically fulfills (cf. appendix A)

$$
\mathrm{Op}_{N}\left(g_{k} g_{k-1} \ldots g_{1}\right) \sim \mathrm{Op}_{N}\left(g_{k}\right) \mathrm{Op}_{N}\left(g_{k-1}\right) \ldots \mathrm{Op}_{N}\left(g_{1}\right), \quad N \rightarrow \infty
$$

With the choice

$$
g(\boldsymbol{z})=\exp (\mathrm{i} \boldsymbol{\sigma} \cdot \boldsymbol{B}(q)) \in \mathrm{SU}(2)
$$

we have $\mathrm{U}_{\mathrm{s}}^{2}=\mathrm{Op}_{N}(g)$ and, thus, we may write $\tilde{\mathrm{U}}(\boldsymbol{\phi})=\mathrm{U}_{\mathrm{t}}(\boldsymbol{\phi}) \mathrm{Op}_{N}(g)$. We now rearrange $\tilde{\mathrm{U}}^{n}(\phi)$ as follows:

$$
\begin{array}{r}
\tilde{\mathrm{U}}^{n}(\phi)=\mathrm{U}_{\mathrm{t}}^{n}(\boldsymbol{\phi})\left(\mathrm{U}_{\mathrm{t}}^{1-n}(\phi) \mathrm{Op}_{N}(g) \mathrm{U}_{\mathrm{t}}^{n-1}(\phi)\right)\left(\mathrm{U}_{\mathrm{t}}^{2-n}(\phi) \mathrm{Op}_{N}(g) \mathrm{U}_{\mathrm{t}}^{n-2}(\phi)\right) \cdots \\
\cdots\left(\mathrm{U}_{\mathrm{t}}^{-1}(\boldsymbol{\phi}) \mathrm{Op}_{N}(g) \mathrm{U}_{\mathrm{t}}(\phi)\right) \mathrm{Op}_{N}(g)
\end{array}
$$


Applying Egorov's theorem (appendix A)

$$
\mathrm{U}_{\mathrm{t}}^{k-n}(\phi) \mathrm{Op}_{N}(g) \mathrm{U}_{\mathrm{t}}^{n-k}(\phi) \sim \mathrm{Op}_{N}\left(g \circ \phi^{n-k}\right), \quad N \rightarrow \infty
$$

to each factor $(k=1, \ldots, n)$ yields

$$
\tilde{\mathrm{U}}^{n}(\phi) \sim \mathrm{U}_{\mathrm{t}}^{n}(\phi) \prod_{k=0}^{n-1} \mathrm{Op}_{N}\left(g \circ \phi^{k}\right), \quad N \rightarrow \infty
$$

where the product is time ordered, i.e.

$$
\prod_{k=0}^{n-1} \mathrm{Op}_{N}\left(g \circ \phi^{k}\right)=\mathrm{Op}_{N}\left(g \circ \phi^{n-1}\right) \mathrm{Op}_{N}\left(g \circ \phi^{n-2}\right) \ldots \mathrm{Op}_{N}(g) .
$$

In order to simplify the notation, it is convenient to set

$$
g_{k}(\boldsymbol{z})=g \circ \boldsymbol{\phi}^{k-1}(\boldsymbol{z}), \quad k=0, \ldots, n,
$$

with the convention that $g_{0}(\boldsymbol{z})=\mathbb{1}_{2}$, and $g_{1}(\boldsymbol{z})=g(\boldsymbol{z})=\exp (\mathrm{i} \boldsymbol{\sigma} \cdot \boldsymbol{B}(q))$. Now eq. (5.8) becomes

$$
\tilde{\mathrm{U}}^{n}(\phi) \sim \mathrm{U}_{\mathrm{t}}^{n}(\phi) \prod_{k=0}^{n} \mathrm{Op}_{N}\left(g_{k}\right), \quad N \rightarrow \infty
$$

In the case when $\phi=A$ is an unperturbed cat map, Egorov's theorem is an identity, not merely an asymptotic relation, and hence eq. (5.11) is an identity too. Due to (5.4) in the semiclassical limit $(N \rightarrow \infty)$ eq. (5.11) becomes

$$
\tilde{\mathrm{U}}^{n}(\phi) \sim \mathrm{U}_{\mathrm{t}}^{n}(\phi) \mathrm{Op}_{N}\left(d_{n}\right),
$$

where

$$
d_{n}(\boldsymbol{z})=\prod_{k=0}^{n} g_{k}(\boldsymbol{z}) \in \mathrm{SU}(2) .
$$

Let $\varphi_{1}, \ldots, \varphi_{N}$ be an orthogonal basis of eigenfunctions of $\mathrm{U}_{N}(\phi)$ with eigenphases $\omega_{j}$,

$$
\mathrm{U}_{N}(\phi) \varphi_{j}=\mathrm{e}^{\mathrm{i} \omega_{j}} \varphi_{j}
$$

Taking the trace of the right-hand side of (5.12) yields

$$
\operatorname{Tr}\left[\mathrm{U}_{\mathrm{t}}^{n}(\phi) \mathrm{Op}_{N}\left(d_{n}\right)\right]=\sum_{j=1}^{N}\left(\varphi_{j}, \mathrm{Op}_{N}\left(\operatorname{tr} d_{n}\right) \varphi_{j}\right) \mathrm{e}^{\mathrm{i} n \omega_{j}}
$$


The leading order term in the semiclassical limit $N \rightarrow \infty$ of the above can be shown to be (cf. appendix B)

$$
\sum_{j=1}^{N}\left(\varphi_{j}, \mathrm{Op}_{N}\left(\operatorname{tr} d_{n}\right) \varphi_{j}\right) \mathrm{e}^{\mathrm{i} n \omega_{j}} \sim \sum_{\boldsymbol{z}_{\mathrm{f}}} \frac{\operatorname{tr} d_{n}\left(\boldsymbol{z}_{\mathrm{f}}\right)}{\sqrt{-R_{\boldsymbol{z}_{\mathrm{f}}}^{(n)}}} \exp \left[2 \pi \mathrm{i} N\left(S_{\boldsymbol{\phi}^{n}}\left(q_{\mathrm{f}}+m_{1}, q_{\mathrm{f}}\right)-m_{2} q_{\mathrm{f}}\right)\right]
$$

Here $\boldsymbol{z}_{\mathrm{f}}$ are fixed points of order $n$ with winding numbers $\left(m_{1}, m_{2}\right)=\boldsymbol{m}$, i.e.

$$
\phi^{n}\left(\boldsymbol{z}_{\mathrm{f}}\right)=\boldsymbol{z}_{\mathrm{f}}+\boldsymbol{m}
$$

Moreover, $R_{\boldsymbol{z}_{\mathrm{f}}}^{(n)}=\operatorname{det}\left(\mathcal{M}_{\boldsymbol{z}_{\mathrm{f}}}^{n}-I\right)$, where $\mathcal{M}_{\boldsymbol{z}_{\mathrm{f}}}^{n}:=\frac{\mathrm{d} \boldsymbol{\phi}^{n}}{\mathrm{~d} \boldsymbol{z}}\left(\boldsymbol{z}_{\mathrm{f}}\right)$ is the monodromy matrix and $S_{\boldsymbol{\phi}^{n}}$ is the generating function of $\phi^{n}$ on the covering plane. We will denote the classical action of the fixed point $\boldsymbol{z}_{\mathrm{f}}$ by

$$
S_{\phi^{n}}\left(\boldsymbol{z}_{\mathrm{f}}\right):=S_{\phi^{n}}\left(q_{\mathrm{f}}+m_{1}, q_{\mathrm{f}}\right)-m_{2} q_{\mathrm{f}}
$$

Thus, in leading semiclassical order $(N \rightarrow \infty)$ we find

$$
\operatorname{Tr}\left[\mathrm{U}^{n}(\boldsymbol{\phi})\right] \sim \sum_{\boldsymbol{z}_{\mathrm{f}}} \frac{\operatorname{tr} d_{n}\left(\boldsymbol{z}_{\mathrm{f}}\right)}{\sqrt{-R_{\boldsymbol{z}_{\mathrm{f}}}^{(n)}}} \exp \left[2 \pi \mathrm{i} N S_{\boldsymbol{\phi}^{n}}\left(\boldsymbol{z}_{\mathrm{f}}\right)\right]
$$

which is the main result of this section. Note that $(\overline{5.19})$ is always a semiclassical expression, even if $\phi=A$ is a cat map. Semiclassical approximations enter in (5.12) and in (5.16) for both the unperturbed and perturbed map. Formula (5.19) shows that the classical orbits are not affected by spin precession and that the spin contribution is represented by the factors $\operatorname{tr} d_{n}\left(\boldsymbol{z}_{\mathrm{f}}\right)$ for each periodic orbit. The respective result in the case of flows was obtained in [12, 13].

\section{Semiclassical analysis of two-point correlations}

We will now apply the trace formula derived in the previous section to the study of spectral two-point correlations, essentially following the line of arguments of [16], giving a new argument concerning equidistribution in $\mathrm{SU}(2)$.

Since $\mathrm{U}$ is invariant under $\tilde{\mathrm{T}}$ with $\tilde{\mathrm{T}}^{2}=-\mathbb{1}_{2 N}$, cf. section 3 , it is easily seen that the eigenvalues must have at least multiplicity two (Kramers' degeneracy). Let us denote by $\alpha_{1}, \ldots, \alpha_{2 N} \in[0,2 \pi)$ the eigenphases of $\mathrm{U}$ with eigenfunctions $\Psi_{j}$, i.e.

$$
\mathrm{U} \Psi_{j}=\mathrm{e}^{\mathrm{i} \alpha_{j}} \Psi_{j}
$$

labelled such that $\alpha_{k}=\alpha_{k+N}$, for $k=1, \ldots, N$. We will then only need to consider correlations between the first $N$ distinct eigenphases $\alpha_{1}, \ldots, \alpha_{N}$. 
In order to measure correlations on the scale of the mean level spacing, we rescale the spectrum by putting

$$
x_{k}=\frac{N}{2 \pi} \alpha_{k}, \quad k=1, \ldots, N .
$$

The two-point correlation density of this sequence is defined as

$$
R_{2}(s, N)=\frac{1}{N} \sum_{k, l=1}^{N} \sum_{m \in \mathbb{Z}} \delta\left(s-\left(x_{k}-x_{l}\right)-N m\right)-1,
$$

and its Fourier transform, the spectral form factor $K_{2}(\tau, N)$, reads

$$
\begin{aligned}
K_{2}(\tau, N) & =\int_{0}^{N} R_{2}(s, N) \mathrm{e}^{2 \pi \mathrm{i} \tau s} \mathrm{~d} s=\frac{1}{N}\left|\sum_{k=1}^{N} \mathrm{e}^{2 \pi \mathrm{i} x_{k} \tau}\right|^{2}-N \delta_{n 0} \\
& =\frac{1}{4 N}\left|\operatorname{Tr}\left(\mathrm{U}^{n}\right)\right|^{2}-N \delta_{n 0} .
\end{aligned}
$$

for $\tau=\frac{n}{N}, n \in \mathbb{Z}$. According to the Bohigas-Giannoni-Schmit conjecture [1], one expects that semiclassically $(N \rightarrow \infty)$ the form factor $K_{2}(\tau, N)$ converges on average to the corresponding CSE form factor. That is, for any smooth and rapidly decaying test function $\phi$, we expect

$$
\lim _{N \rightarrow \infty} \sum_{n \in \mathbb{Z}} K_{2}\left(\frac{n}{N}, N\right) \phi\left(\frac{n}{N}\right)=\int_{\mathbb{R}} K_{2}^{\mathrm{CSE}}(\tau) \phi(\tau) \mathrm{d} \tau
$$

with 25]

$$
K_{2}^{\mathrm{CSE}}(\tau)= \begin{cases}\frac{1}{2}|\tau|-\frac{1}{4}|\tau| \log |1-\tau| & \text { for }|\tau| \leq 2 \\ 1 & \text { for }|\tau|>2 .\end{cases}
$$

This statement implies that also the pair correlation density $R_{2}(s, N)$ converges (on average) to the corresponding CSE density.

It seems to be extremely difficult to prove rel. (6.5) with present techniques. Here, we will aim at understanding the asymptotics of the form factor for small values of $\tau$, as $N \rightarrow \infty$, in the regime governed by the diagonal approximation [14, 15]. For the diagonal approximation to hold, we will assume in the following that there are no systematic degeneracies in the classical actions of the map under consideration. This is true for generic perturbed cat maps $\phi$, but not for the original cat maps $A$, where actions are in fact highly degenerate [23, 26].

By virtue of our trace formula (5.19) we have, for $\tau=\frac{n}{N} \neq 0, N \rightarrow \infty$,

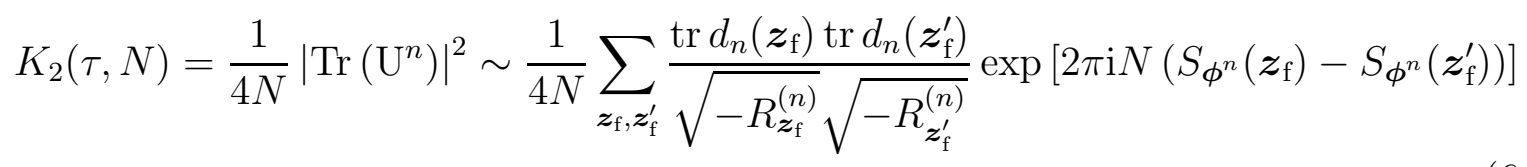


(recall $\operatorname{tr} d_{n}\left(\boldsymbol{z}_{\mathrm{f}}^{\prime}\right)=\overline{\operatorname{tr} d_{n}\left(\boldsymbol{z}_{\mathrm{f}}^{\prime}\right)}$ for $\left.d_{n} \in \mathrm{SU}(2)\right)$, where the sum extends over all fixed points of order $n$. Since for $S_{\boldsymbol{\phi}^{n}}\left(\boldsymbol{z}_{\mathrm{f}}\right) \neq S_{\boldsymbol{\phi}^{n}}\left(\boldsymbol{z}_{\mathrm{f}}^{\prime}\right)$, cf. (5.18), the exponential in (6.7) shows rapid oscillations as $N \rightarrow \infty$, we assume that in the combined limit

$$
\tau \rightarrow 0, \quad N \rightarrow \infty, \quad n=\tau N \rightarrow \infty
$$

the double sum in (6.7) is dominated by the diagonal terms [14, 15, i.e. by the terms with $S_{\boldsymbol{\phi}^{n}}\left(\boldsymbol{z}_{\mathrm{f}}\right)=S_{\boldsymbol{\phi}^{n}}\left(\boldsymbol{z}_{\mathrm{f}}^{\prime}\right)$. We know that

$$
S_{\boldsymbol{\phi}^{n}}\left(\boldsymbol{z}_{\mathrm{f}}\right)=S_{\boldsymbol{\phi}^{n}}\left(\boldsymbol{z}_{\mathrm{f}}^{\prime}\right), \quad R_{\boldsymbol{z}_{\mathrm{f}}}^{(n)}=R_{\boldsymbol{z}_{\mathrm{f}}^{\prime}}^{(n)}, \quad \operatorname{tr} d_{n}\left(\boldsymbol{z}_{\mathrm{f}}\right)=\operatorname{tr} d_{n}\left(\boldsymbol{z}_{\mathrm{f}}^{\prime}\right)
$$

for all points $\boldsymbol{z}_{\mathrm{f}}^{\prime}$ along the periodic orbit (compare section 5),

$$
\boldsymbol{z}_{\mathrm{f}}^{\prime}=\boldsymbol{\phi}\left(\boldsymbol{z}_{\mathrm{f}}\right), \ldots, \phi^{n-1}\left(\boldsymbol{z}_{\mathrm{f}}\right), \phi^{n}\left(\boldsymbol{z}_{\mathrm{f}}\right)=\boldsymbol{z}_{\mathrm{f}} .
$$

Thus, we find $n^{\#}$ degenerate actions for every orbit of period $n, n^{\#}$ denoting the primitive period, i.e. $n=k n^{\#}, k \in \mathbb{N}$. Furthermore, since our system is invariant under (nonconventional) time-reversal $\tilde{\mathrm{T}}$, we have

$$
S_{\phi^{n}}\left(\boldsymbol{z}_{\mathrm{f}}\right)=S_{\boldsymbol{\phi}^{n}}\left(\tilde{\boldsymbol{\tau}}\left(\boldsymbol{z}_{\mathrm{f}}\right)\right), \quad R_{\boldsymbol{z}_{\mathrm{f}}}^{(n)}=R_{\tilde{\boldsymbol{\tau}}\left(\boldsymbol{z}_{\mathrm{f}}\right)}^{(n)}, \quad \operatorname{tr} d_{n}\left(\boldsymbol{z}_{\mathrm{f}}\right)=\operatorname{tr} d_{n}\left(\tilde{\boldsymbol{\tau}}\left(\boldsymbol{z}_{\mathrm{f}}\right)\right) .
$$

Neglecting self-retracing orbits in the limit $n \rightarrow \infty$ this results in an additional factor of 2 in the diagonal approximation. Note that if the classical map $\phi$ has a further symmetry, e.g. inversion $\boldsymbol{\pi}$ (3.2), we also have $S_{\boldsymbol{\phi}^{n}}\left(\boldsymbol{\pi}\left(\boldsymbol{z}_{\mathrm{f}}\right)\right)=S_{\boldsymbol{\phi}^{n}}\left(\boldsymbol{z}_{\mathrm{f}}\right)$. However, since in general tr $d_{n}\left(\boldsymbol{z}_{\mathrm{f}}\right)$ and $\operatorname{tr} d_{n}\left(\boldsymbol{\pi}\left(\boldsymbol{z}_{\mathrm{f}}\right)\right)$ become uncorrelated for large $n$, we will neglect cross correlations of these terms. We therefore conclude that in the combined limit (6.8) the form factor can be approximated by

$$
K_{2}(\tau, N) \sim \frac{2 n}{4 N} \sum_{\boldsymbol{z}_{\mathrm{f}}} \frac{\left(\operatorname{tr} d_{n}\left(\boldsymbol{z}_{\mathrm{f}}\right)\right)^{2}}{-R_{\boldsymbol{z}_{\mathrm{f}}}^{(n)}} .
$$

It is well known [27] that the number of fixed points of Anosov maps grows, on average, like $\sim \mathrm{e}^{h n}$ for $n$ large, where $h$ denotes the topological entropy. What is more, the fixed points become equidistributed in phase space $\mathbb{T}^{2}$ [14, 27], in the sense that for any smooth test function $a(\boldsymbol{z})$, we have for $n$ large

$$
\left\langle\sum_{\boldsymbol{z}_{\mathrm{f}}} \frac{a\left(\boldsymbol{z}_{\mathrm{f}}\right)}{-R_{\boldsymbol{z}_{\mathrm{f}}}^{(n)}}\right\rangle_{n} \sim \int_{\mathbb{T}^{2}} a(\boldsymbol{z}) \mathrm{d} z,
$$

where the average $\langle\ldots\rangle_{n}$ is some linear mean over an interval about $n$ whose size grows to $\infty$ as $n \rightarrow \infty$. Let us ignore the fact that $d_{n}(\boldsymbol{z})$ depends on $n$, since, as we shall justify below, it in fact converges on average to a constant independent of $n$. Hence by virtue of (6.13)

$$
\left\langle\sum_{\boldsymbol{z}_{\mathrm{f}}} \frac{\left(\operatorname{tr} d_{n}\left(\boldsymbol{z}_{\mathrm{f}}\right)\right)^{2}}{-R_{\boldsymbol{z}_{\mathrm{f}}}^{(n)}}\right\rangle_{n} \sim \int_{\mathbb{T}^{2}}\left\langle\left(\operatorname{tr} d_{n}(\boldsymbol{z})\right)^{2}\right\rangle_{n} \mathrm{~d} z .
$$


In the semiclassical limit the quantum dynamics reduces to the skew product dynamical system [16

$$
\begin{aligned}
\boldsymbol{Y}: \mathbb{T}^{2} \times \mathrm{SU}(2) & \rightarrow \mathbb{T}^{2} \times \mathrm{SU}(2) \\
(\boldsymbol{z}, g) & \mapsto\left(\boldsymbol{\phi}(\boldsymbol{z}), g_{1}(\boldsymbol{z}) g\right)
\end{aligned}
$$

with $g_{1}(\boldsymbol{z})=\exp (\mathrm{i} \boldsymbol{\sigma} \cdot \boldsymbol{B}(q))$. The $n$th iterate is then given by

$$
\boldsymbol{Y}^{n}(\boldsymbol{z}, g)=\left(\boldsymbol{\phi}^{n}(\boldsymbol{z}), d_{n}(\boldsymbol{z}) g\right)
$$

The right-hand-side of (6.14) can be viewed as a sequence of probability measures

$$
\nu_{n}(F)=\int_{\mathbb{T}^{2}}\left\langle F\left(\boldsymbol{Y}^{n}\left(\boldsymbol{z}, \mathbb{1}_{2}\right)\right)\right\rangle_{n} \mathrm{~d} z
$$

if we put $F(\boldsymbol{z}, g)=(\operatorname{tr} g)^{2}$. For $F$ bounded on $\mathbb{T}^{2} \times \mathrm{SU}(2)$, this sequence is contained in a compact space of probability measures, hence we find a convergent subsequence $n_{j}$ with some limit measure $\mu$, i.e.

$$
\lim _{j \rightarrow \infty} \nu_{n_{j}}(F)=\mu(F)
$$

Due to the $\langle\ldots\rangle_{n}$ average we have, for $n$ large,

$$
\nu_{n}\left(F \circ \boldsymbol{Y}^{r}\right) \sim \nu_{n}(F)
$$

for any fixed integer $r$. Moreover, (with the substitution $w=\phi(z)$ ) we have

$$
\begin{aligned}
\nu_{n}(F \circ \boldsymbol{Y}) & =\int_{\mathbb{T}^{2}}\left\langle F\left(\phi^{n+1}(z), d_{n+1}(z)\right)\right\rangle_{n} \mathrm{~d} z=\int_{\mathbb{T}^{2}}\left\langle F\left(\phi^{n}(w), d_{n+1}\left(\phi^{-1}(w)\right)\right)\right\rangle_{n} \mathrm{~d} w \\
& =\int_{\mathbb{T}^{2}}\left\langle F\left(\phi^{n}(w), d_{n}(w) g_{1}\left(\phi^{-1}(w)\right)\right)\right\rangle_{n} \mathrm{~d} w=\nu_{n}\left(F \circ \gamma_{1}\right)
\end{aligned}
$$

where $\gamma_{1}$ is defined by

$$
\gamma_{1}:(z, g) \mapsto\left(z, g g_{1}\left(\phi^{-1}(z)\right)\right)
$$

Therefore, we have $\nu_{n}\left(F \circ \gamma_{1}\right) \sim \nu_{n}(F)$, and similarly, since

$$
\int_{\mathbb{T}^{2}} F\left(\phi^{n+r}(\boldsymbol{z}), d_{n+r}(\boldsymbol{z})\right) \mathrm{d} z=\int_{\mathbb{T}^{2}} F\left(\phi^{n}(\boldsymbol{z}), d_{n}(\boldsymbol{z}) d_{r}\left(\boldsymbol{\phi}^{-r}(\boldsymbol{z})\right)\right) \mathrm{d} z
$$

for any fixed $r \in \mathbb{Z}$ we obtain

$$
\nu_{n}\left(F \circ \gamma_{r}\right) \sim \nu_{n}(F)
$$


with $\boldsymbol{\gamma}_{r}:(\boldsymbol{z}, g) \mapsto\left(\boldsymbol{z}, g d_{r}\left(\boldsymbol{\phi}^{-r}(\boldsymbol{z})\right)\right)$. Thus, we conclude that the limit measure $\mu$ itself must be invariant, i.e. $\mu\left(F \circ \tilde{\gamma}_{r}\right)=\mu(F)$, for all $r \in \mathbb{Z}$. Clearly, since $\mu$ is invariant under $\gamma_{r}$, it is also invariant under $\gamma_{r}^{-1}$ and in particular we obtain the relation

$$
\mu\left(F \circ \tilde{\gamma}_{r}\right) \sim \mu(F) \quad \text { with } \quad \tilde{\gamma}_{r}:=\gamma_{r} \circ \gamma_{r-1}^{-1}:(z, g) \mapsto\left(z, g g_{1}\left(\phi^{-r}(z)\right)\right) .
$$

So if, for almost all $\boldsymbol{z} \in \mathbb{T}^{2}$, we find a set $V$ of integers such that the group generated by $\left\{g_{1}\left(\boldsymbol{\phi}^{-\nu}(\boldsymbol{z})\right)\right\}_{\nu \in V}$ is dense in $\mathrm{SU}(2)$, then the action of that group is uniquely ergodic on $\mathrm{SU}(2)$ (see 28 for more details and references on equidistribution on $\mathrm{SU}(2)$ ), and we have that $d \mu=d z d g$, where $d g$ denotes Haar measure. Clearly it is always possible to satisfy the above condition for any generic choice of $g_{1}$, i.e. for any generic magnetic field $\boldsymbol{B}(q)$, cf. section 3 .

Since the limit $\mu$ of every converging subsequence is unique, in fact every subsequence converges to $\mu$. That is

$$
\lim _{n \rightarrow \infty} \int_{\mathbb{T}^{2}}\left\langle F\left(\boldsymbol{Y}^{n}\left(\boldsymbol{z}, \mathbb{1}_{2}\right)\right)\right\rangle_{n} \mathrm{~d} z=\int_{\mathbb{T}^{2}} \int_{\mathrm{SU}(2)} F(\boldsymbol{z}, g) \mathrm{d} z \mathrm{~d} g .
$$

In our case $F(\boldsymbol{z}, g)=(\operatorname{tr} g)^{2}$, and by the character formula 29]

$$
\int_{\mathbb{T}^{2}} \int_{\mathrm{SU}(2)} F(\boldsymbol{z}, g) \mathrm{d} z \mathrm{~d} g=\int_{\mathrm{SU}(2)}(\operatorname{tr} g)^{2} \mathrm{~d} g=1 .
$$

Therefore, for generic magnetic fields $\boldsymbol{B}(q)$, the asymptotics of the form factor at small $\tau$ is in the diagonal approximation given by

$$
K_{2}(\tau, N) \sim \frac{1}{2} \tau
$$

which is identical to the small $\tau$ asymptotics of the CSE form factor (6.6).

\section{$7 \quad$ Piecewise constant magnetic fields}

As we have seen in the previous section, the diagonal approximation works when the spin precession becomes equidistributed in $\mathrm{SU}(2)$. It is quite remarkable that this is not a necessary condition. Suppose for instance that the magnetic field is piecewise constant, such that $g_{1}\left(\boldsymbol{\phi}^{1}(\boldsymbol{z})\right), g_{1}\left(\boldsymbol{\phi}^{2}(\boldsymbol{z})\right), \ldots$ only takes values in a discrete set in $\mathrm{SU}(2)$. In this case the equidistribution theorem (6.25) holds with $\mathrm{SU}(2)$ replaced by $\Gamma$, where $\Gamma$ is the finite subgroup generated by $\left\{g_{1}\left(\boldsymbol{\phi}^{r} \boldsymbol{z}\right)\right\}_{r}$,

$$
\lim _{n \rightarrow \infty} \int_{\mathbb{T}^{2}}\left\langle F\left(\boldsymbol{Y}^{n}\left(\boldsymbol{z}, \mathbb{1}_{2}\right)\right)\right\rangle_{n} \mathrm{~d} z=\frac{1}{|\Gamma|} \sum_{g \in \Gamma} \int_{\mathbb{T}^{2}} F(\boldsymbol{z}, g) \mathrm{d} z,
$$

where $|\Gamma|$ is the order of $\Gamma$. Now

$$
K_{2}(\tau, N) \sim \frac{1}{2} \tau \frac{1}{|\Gamma|} \sum_{g \in \Gamma}(\operatorname{tr} g)^{2}
$$


If the representation of $\Gamma$ in $\mathrm{SU}(2)$ is irreducible, we have as a consequence of Schur's Lemma (see, e.g., 229] for details)

$$
\frac{1}{|\Gamma|} \sum_{g \in \Gamma}(\operatorname{tr} g)^{2}=1,
$$

as above. Irreducibility is guaranteed by our assumption in section 3 that there be no vector $\boldsymbol{r} \neq 0$ such that $\boldsymbol{r} \cdot \boldsymbol{B}(q)=0$ for all $q$. Hence we have again

$$
K_{2}(\tau, N) \sim \frac{1}{2} \tau \sim K_{2}^{\mathrm{CSE}}(\tau),
$$

for $\tau$ small.

Let us complement this argument by a numerical experiment. We choose the magnetic field

$$
\boldsymbol{B}(q)= \begin{cases}(\pi, 0,0) & \text { if } 0 \leq q<1 / 6 \\ (0, \pi, 0) & \text { if } 1 / 6 \leq q<1 / 3 \\ (0,0, \pi) & \text { if } 1 / 3 \leq q<1 / 2 \\ (0,0,0) & \text { if } q=1 / 2\end{cases}
$$

The symmetry constraint $\boldsymbol{B}(1-q)=-\boldsymbol{B}(q)$ determines the magnetic field in the interval $1 / 2<q<1$. We also require periodicity, i.e. $\boldsymbol{B}(q+m)=\boldsymbol{B}(q), m \in \mathbb{Z}$. This particular choice leads to the discrete group of Hamilton's quaternions,

$$
\Gamma=\left\{ \pm \mathbb{1}_{2}, \pm \mathrm{i} \sigma_{x}, \pm \mathrm{i} \sigma_{y}, \pm \mathrm{i} \sigma_{z}\right\} .
$$

The quantum maps $\mathrm{U}(A)$ and $\mathrm{U}(\phi)$ defined in (4.4) can also be easily diagonalized with the magnetic field given by (7.5). The statistics are clearly CSE as shown in fig. [3. This outcome could be expected for generic Anosov maps, since the diagonal approximation for the form factor agrees, at small argument, with the random matrix prediction.

In the case of the unperturbed cat map, however, we cannot proceed as in section 6 due to the exponentially large number of fixed points sharing the same classical action 30, 23. Remarkably, we still observe CSE statistics (fig. 3). Similar peculiarities are found for arithmetic triangles with non-conventional boundary conditions [31, non-arithmetic Hecke triangles [32] and hyperbolic tetrahedra [33].

\section{Acknowledgment}

We gratefully acknowledge helpful discussions with Jens Bolte, Grischa Haag, Jon Keating and Jonathan Robbins. S K was partly supported by Deutscher Akademischer Austauschdienst (DAAD) under grant no. D/99/02553 and by Deutsche Forschungsgemeinschaft (DFG) under contract no. STE 241/10-1. F M was supported by a Royal Society Dorothy Hodgkin Fellowship during the period when this research was completed. 


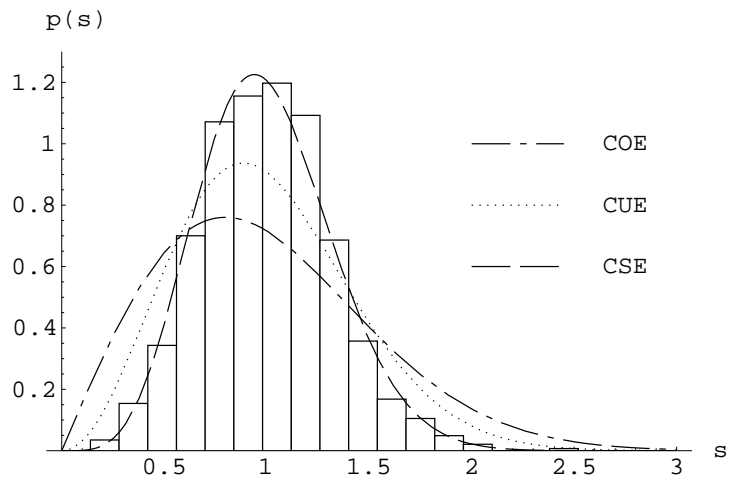

(a) Spacing distribution of $\mathrm{U}(A)$ as defined in (4.4)

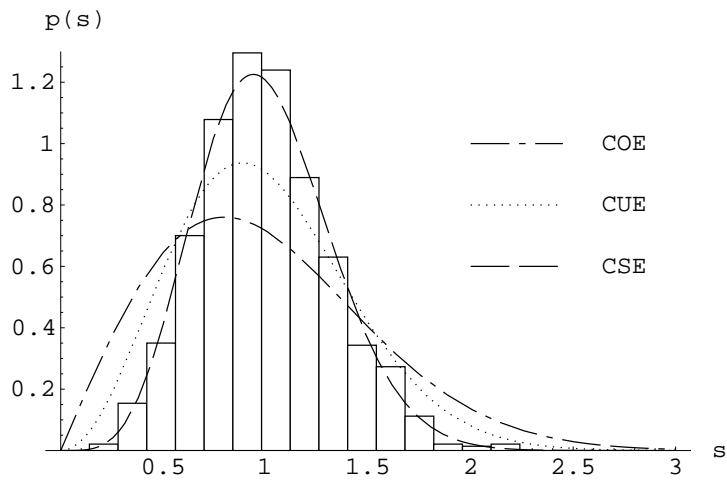

(c) Spacing distribution of $\mathrm{U}(\phi)$ as defined in (4.4). The perturbation parameter is $\mathrm{k}=0.32$.

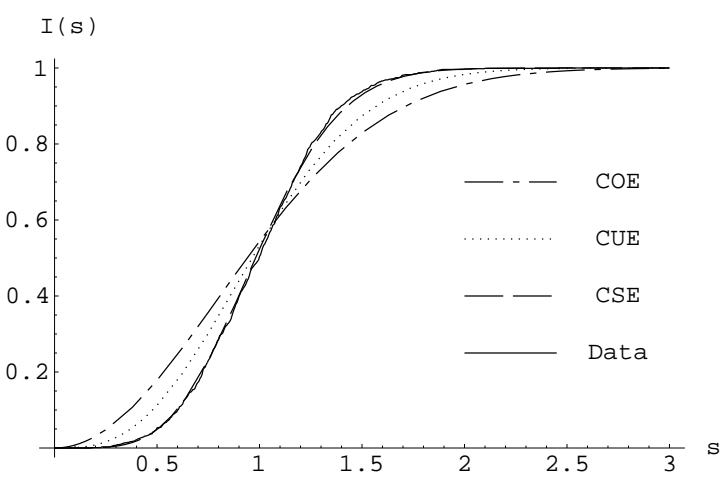

(b) Cumulative spacing distribution of $\mathrm{U}(A)$ as defined in (4.4)

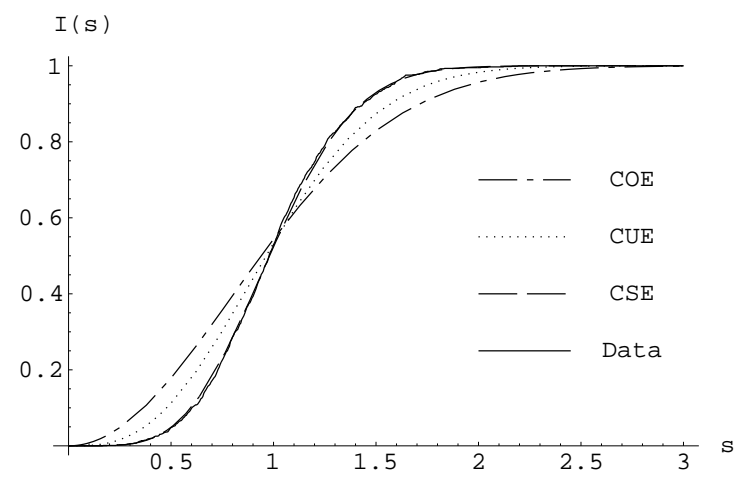

(d) Cumulative spacing distribution of $\mathrm{U}(\phi)$ as defined with $\mathrm{k}=0.32$.

Figure 3: Local spectral statistics of the quantum cat map (above) and of its perturbation (below) when the spin precession is caused by the magnetic field (7.5)). The number of eigenangles $N$ is 1021 . 


\section{A Quantum mechanics on the torus}

We briefly review quantum mechanics of systems whose classical phase space is the torus $\mathbb{T}^{2}$. For more details see [17, 34, 35].

Because of the topology of the torus, quantum states are taken to be periodic in both position and momentum representation, i.e.

$$
\psi\left(q+m_{1}\right)=\psi(q), \quad \hat{\psi}\left(p+m_{2}\right)=\hat{\psi}(p), \quad m_{1}, m_{2} \in \mathbb{Z}
$$

where

$$
\hat{\psi}(p)=\frac{1}{\sqrt{2 \pi \hbar}} \int_{-\infty}^{+\infty} \psi(q) \mathrm{e}^{-\frac{\mathrm{i}}{\hbar} q p} \mathrm{~d} q .
$$

The periodicity of the wavefunction in both bases has two important consequences. Firstly, both $\psi(q)$ and $\hat{\psi}(p)$ are superpositions of delta functions supported on the lattices points $q=2 \pi \hbar Q$ and $p=2 \pi \hbar P$ respectively, where $Q, P \in \mathbb{Z}$, i.e.

$$
\psi(q)=\sum_{m \in \mathbb{Z}} \sum_{Q=0}^{N-1} \Psi(Q) \delta\left(q-\frac{Q}{N}+m\right)
$$

with $\Psi(Q+N)=\Psi(Q)$. Secondly, $2 \pi \hbar$ must be an inverse integer, i.e. $N=1 / 2 \pi \hbar$. It follows that the Hilbert space may be identified with the $N$-dimensional vector space $\mathcal{H}_{N}$ with inner product

$$
(\Phi, \Psi)=\frac{1}{N} \sum_{Q \bmod N} \bar{\Phi}(Q) \Psi(Q) .
$$

In order to quantize observables $f \in C^{\infty}\left(\mathbb{T}^{2}\right)$ we need to introduce the translation operators

$$
\begin{aligned}
& t_{1} \Phi(Q)=\Phi(Q+1) \\
& t_{2} \Phi(Q)=\Phi(Q) \exp \left(\frac{2 \pi \mathrm{i}}{N} Q\right),
\end{aligned}
$$

which may be viewed as the exponentials of the usual differentiation and multiplication operators on the real line. For any $m, n \in \mathbb{Z}$, we have the following commutation relation

$$
t_{1}^{m} t_{2}^{n}=\exp \left(\frac{2 \pi \mathrm{i}}{N} m n\right) t_{2}^{n} t_{1}^{m}
$$

Note that $t_{1}^{N}=t_{2}^{N}=\mathbb{1}_{N}$. The Weyl-Heisenberg operators are defined by

$$
T_{N}(\boldsymbol{n})=\exp \left(\frac{\pi \mathrm{i}}{N} n_{1} n_{2}\right) t_{2}^{n_{2}} t_{1}^{n_{1}}
$$


where $\boldsymbol{n}=\left(n_{1}, n_{2}\right)$. We then have the following multiplication rule

$$
T_{N}(\boldsymbol{m}) T_{N}(\boldsymbol{n})=\exp \left(\frac{\pi \mathrm{i}}{N} \omega(\boldsymbol{m}, \boldsymbol{n})\right) T_{N}(\boldsymbol{m}+\boldsymbol{n}),
$$

where $\omega(\boldsymbol{m}, \boldsymbol{n})=m_{1} n_{2}-m_{2} n_{1}$ is the standard symplectic form.

Let $f \in C^{\infty}\left(\mathbb{T}^{2}\right)$ be a classical observable on $\mathbb{T}^{2}$ whose Fourier series is given by

$$
f(\boldsymbol{z})=\sum_{\boldsymbol{m} \in \mathbb{Z}^{2}} \hat{f}_{\boldsymbol{m}} \mathrm{e}^{2 \pi \mathrm{i} \boldsymbol{z} \cdot \boldsymbol{m}}, \quad \boldsymbol{z}=(p, q) \in \mathbb{T}^{2} .
$$

The Weyl quantization of $f$ is defined as

$$
\mathrm{Op}_{N}(f)=\sum_{\boldsymbol{m} \in \mathbb{Z}^{2}} \hat{f}_{\boldsymbol{m}} T_{N}(\boldsymbol{m})
$$

Since $\mathrm{Op}_{N}\left(f^{*}\right)=\mathrm{Op}_{N}(f)^{\dagger}, \mathrm{Op}_{N}(f)$ is Hermitian if and only if $f(\boldsymbol{z})$ is real.

Semiclassically, quantum observables commute, which can be easily seen by expanding the exponential in (A.8). More precisely, let $f, g \in C^{\infty}\left(\mathbb{T}^{2}\right)$, then

$$
\mathrm{Op}_{N}(f) \mathrm{Op}_{N}(g) \sim \mathrm{Op}_{N}(f g), \quad N \rightarrow \infty .
$$

If $f$ and $g$ depend only on either $p$ or $q$, the above relation is an identity for each $N$. The quantization of classical matrix-valued observables is analogous to the case of scalar functions. Let $g(\boldsymbol{z})$ be a $2 \times 2$ matrix such that $g_{j k} \in C^{\infty}\left(\mathbb{T}^{2}\right)$. We define its Weyl quantization as

$$
\mathrm{Op}_{N}(g)=\left(\begin{array}{ll}
\mathrm{Op}_{N}\left(g_{11}\right) & \mathrm{Op}_{N}\left(g_{12}\right) \\
\operatorname{Op}_{N}\left(g_{21}\right) & \mathrm{Op}_{N}\left(g_{22}\right)
\end{array}\right)
$$

From (A.11) it follows that

$$
\mathrm{Op}_{N}\left(g_{k} \ldots g_{2} g_{1}\right) \sim \mathrm{Op}_{N}\left(g_{k}\right) \ldots \mathrm{Op}_{N}\left(g_{2}\right) \mathrm{Op}_{N}\left(g_{1}\right), \quad N \rightarrow \infty .
$$

Clearly, we have

$$
\mathrm{Op}_{N}\left(g^{\dagger}\right)=\mathrm{Op}_{N}(g)^{\dagger} \text {. }
$$

If $g(\boldsymbol{z})$ is unitary, combining (A.13) and (A.14) yields

$$
\mathrm{Op}_{N}(g) \mathrm{Op}_{N}(g)^{\dagger} \sim \mathbb{1}_{2 N}, \quad N \rightarrow \infty
$$

that is $\mathrm{Op}_{N}(g)$ is only semiclassically unitary.

We can now formulate a version of Egorov's theorem [36] for matrix valued observables. Let

$$
\mathrm{U}_{\mathrm{t}}(\phi)=\left(\begin{array}{cc}
\mathrm{U}_{N}(\phi) & 0 \\
0 & \mathrm{U}_{N}(\phi)
\end{array}\right)
$$


where $\mathrm{U}_{N}(\phi)$ is the quantum propagator of an Anosov map $\phi$. We have

$$
\begin{aligned}
\mathrm{U}_{\mathrm{t}}^{-1}(\boldsymbol{\phi}) \mathrm{Op}_{N}(g) \mathrm{U}_{\mathrm{t}}(\boldsymbol{\phi}) & =\left(\begin{array}{ll}
\mathrm{U}_{N}(\boldsymbol{\phi})^{-1} \mathrm{Op}_{N}\left(g_{11}\right) \mathrm{U}_{N}(\boldsymbol{\phi}) & \mathrm{U}_{N}(\boldsymbol{\phi})^{-1} \mathrm{Op}_{N}\left(g_{12}\right) \mathrm{U}_{N}(\boldsymbol{\phi}) \\
\mathrm{U}_{N}(\boldsymbol{\phi})^{-1} \mathrm{Op}_{N}\left(g_{21}\right) \mathrm{U}_{N}(\boldsymbol{\phi}) & \mathrm{U}_{N}(\boldsymbol{\phi})^{-1} \mathrm{Op}_{N}\left(g_{22}\right) \mathrm{U}_{N}(\boldsymbol{\phi})
\end{array}\right) \\
& \sim\left(\begin{array}{ll}
\mathrm{Op}_{N}\left(g_{11} \circ \boldsymbol{\phi}\right) & \mathrm{Op}_{N}\left(g_{12} \circ \boldsymbol{\phi}\right) \\
\mathrm{Op}_{N}\left(g_{21} \circ \boldsymbol{\phi}\right) & \mathrm{Op}_{N}\left(g_{22} \circ \boldsymbol{\phi}\right)
\end{array}\right)=\mathrm{Op}_{N}(g \circ \boldsymbol{\phi}),
\end{aligned}
$$

as $N \rightarrow \infty$. For cat maps this relation is an identity.

\section{B Trace formula for matrix elements}

The following trace formula for matrix elements of quantum observables is a well known generalization of Gutzwiller's trace formula [37, 38, 39]. We will here present its derivation in the case of general Anosov maps $\phi$ on the torus $\mathbb{T}^{2}$, which is particularly clean and simple. The formula is needed in section 5 .

Suppose $\varphi_{1}, \ldots, \varphi_{N}$ is an orthogonal basis of eigenfunctions of $\mathrm{U}_{N}(\phi)$ with eigenphases $\omega_{j}$,

$$
\mathrm{U}_{N}(\phi) \varphi_{j}=\mathrm{e}^{\mathrm{i} \omega_{j}} \varphi_{j}
$$

Claim: For any smooth classical observable $f \in \mathbb{C}^{\infty}\left(\mathbb{T}^{2}\right)$ and $n$ fixed we have in the semiclassical limit $(N \rightarrow \infty)$

$$
\sum_{j=1}^{N}\left(\varphi_{j}, \mathrm{Op}_{N}(f) \varphi_{j}\right) \mathrm{e}^{\mathrm{i} n \omega_{j}} \sim \sum_{\boldsymbol{z}_{\mathrm{f}}} \frac{f\left(\boldsymbol{z}_{\mathrm{f}}\right)}{\sqrt{-R_{\boldsymbol{z}_{\mathrm{f}}}^{(n)}}} \exp \left[2 \pi \mathrm{i} N\left(S_{\boldsymbol{\phi}^{n}}\left(q_{\mathrm{f}}+m_{1}, q_{\mathrm{f}}\right)-m_{2} q_{\mathrm{f}}\right)\right]
$$

where $\left(m_{1}, m_{2}\right)=\boldsymbol{m}$ are the winding numbers and $\boldsymbol{z}_{\mathrm{f}}$ are the fixed points of order $n$, i.e.

$$
\phi^{n}\left(\boldsymbol{z}_{\mathrm{f}}\right)=\boldsymbol{z}_{\mathrm{f}}+\boldsymbol{m}
$$

Furthermore, $R_{\boldsymbol{z}_{\mathrm{f}}}^{(n)}=\operatorname{det}\left(\mathcal{M}_{\boldsymbol{z}_{\mathrm{f}}}^{n}-I\right)$, where $\mathcal{M}_{\boldsymbol{z}_{\mathrm{f}}}^{n}=\frac{\mathrm{d} \boldsymbol{\phi}}{\mathrm{d} \boldsymbol{z}}\left(\boldsymbol{z}_{\mathrm{f}}\right)$ denotes the monodromy matrix and $S_{\phi^{n}}$ generates the classical map $\phi^{n}$.

The left-hand-side of $(\mathbb{B . 2})$ is of course the trace of $\mathrm{U}_{N}^{n}(\phi) \mathrm{Op}_{N}(f)$. Since

$$
\mathrm{Op}_{N}(f)=\sum_{\boldsymbol{\mu} \in \mathbb{Z}^{2}} \hat{f}_{\boldsymbol{\mu}} T_{N}(\boldsymbol{\mu})
$$

and because $f \in C^{\infty}\left(\mathbb{T}^{2}\right)$ implies that the coefficients $\hat{f}_{\boldsymbol{\mu}}$ are rapidly decreasing, we may without loss of generality consider only the matrix elements $\mathrm{U}_{N}^{n}(\boldsymbol{\phi}) T_{N}(\boldsymbol{\mu})$. For $\nu=0, \ldots, N-1$ the functions

$$
\delta_{\nu}(Q)= \begin{cases}\sqrt{N} & (Q=\nu \bmod N) \\ 0 & (Q \neq \nu \bmod N)\end{cases}
$$


form an orthonormal basis of the Hilbert space $\mathcal{H}_{N}$. Hence we may write

$$
\begin{aligned}
\operatorname{Tr}\left[\mathrm{U}_{N}^{n}(\phi) T_{N}(\boldsymbol{\mu})\right] & =\sum_{\nu \bmod N}\left(\delta_{\nu}, \mathrm{U}_{N}^{n}(\phi) T_{N}(\boldsymbol{\mu}) \delta_{\nu}\right) \\
& =\sum_{\nu \bmod N} \exp \left(\frac{\pi \mathrm{i}}{N} \mu_{1} \mu_{2}\right) \exp \left(\frac{2 \pi \mathrm{i}}{N} \mu_{2}\left(\nu-\mu_{1}\right)\right)\left(\delta_{\nu}, \mathrm{U}_{N}^{n}(\phi) \delta_{\nu-\mu_{1}}\right) \\
& \sim \sum_{\nu \bmod N} \exp \left(\frac{2 \pi \mathrm{i}}{N} \mu_{2} \nu\right)\left(\delta_{\nu}, \mathrm{U}_{N}^{n}(\phi) \delta_{\nu-\mu_{1}}\right)
\end{aligned}
$$

for $N$ large and $\|\boldsymbol{\mu}\|<N^{1 / 2-\delta}, \delta>0$. As we shall justify below any smooth observable can be well approximated with a suitable cut-off in $\boldsymbol{\mu}$. In the semiclassical limit the matrix elements of $\mathrm{U}_{N}^{n}(\phi)$ are given by 18

$$
\left(\delta_{\nu}, \mathrm{U}_{N}^{n}(\phi) \delta_{\nu^{\prime}}\right) \sim \frac{1}{M_{n}} \sum_{m_{1}=0}^{M_{n}} D\left(\frac{\nu}{N}+m_{1}, \frac{\nu^{\prime}}{N}\right) \exp \left[2 \pi \mathrm{i} N S_{\phi^{n}}\left(\frac{\nu}{N}+m_{1}, \frac{\nu^{\prime}}{N}\right)\right],
$$

where

$$
D\left(q_{2}, q_{1}\right)=\left(\frac{\mathrm{i}}{N} \frac{\partial^{2} S_{\phi^{n}}\left(q_{2}, q_{1}\right)}{\partial q_{2} \partial q_{1}}\right)^{1 / 2},
$$

$S_{\phi^{n}}\left(q_{2}, q_{1}\right)$ is the classical action of the lift of $\phi^{n}$ on the covering plane [10], and $M_{n}$ depends on the unperturbed map and on $n$.

Inserting (B.7) into (B.6) and applying the Poisson summation formula yields

$$
\begin{aligned}
\operatorname{Tr}\left[\mathrm{U}_{N}^{n}(\boldsymbol{\phi}) T_{N}(\boldsymbol{\mu})\right] \sim & \frac{1}{M_{n}} \sum_{m_{1}=0}^{M_{n}} \sum_{m_{2} \in \mathbb{Z}} N \int_{-\epsilon}^{1-\epsilon} D\left(q+m_{1}, q-\frac{\mu_{1}}{N}\right) \times \\
& \times \exp \left[2 \pi \mathrm{i} N\left(S_{\phi^{n}}\left(q+m_{1}, q-\frac{\mu_{1}}{N}\right)-m_{2} q\right)\right] \exp \left(2 \pi \mathrm{i} \mu_{2} q\right) \mathrm{d} q
\end{aligned}
$$

In leading order $(N \rightarrow \infty)$ we have, uniformly for all $\boldsymbol{\mu}$ with $\|\boldsymbol{\mu}\| \leq N^{1 / 2-\delta}$,

$$
D\left(q+m_{1}, q-\frac{\mu_{1}}{N}\right) \sim D\left(q+m_{1}, q\right)
$$

and

$$
\begin{aligned}
S_{\phi^{n}}\left(q+m_{1}, q-\frac{\mu_{1}}{N}\right) & \sim S_{\phi^{n}}\left(q+m_{1}, q\right)-\left.\frac{\partial S_{\phi^{n}}\left(q+m_{1}, q^{\prime}\right)}{\partial q^{\prime}}\right|_{q^{\prime}=q} \frac{\mu_{1}}{N} \\
& \sim S_{\phi^{n}}\left(q+m_{1}, q\right)+p(q) \frac{\mu_{1}}{N}
\end{aligned}
$$

Inserting (B.10) into (B.9) gives

$$
\begin{aligned}
\operatorname{Tr}\left[\mathrm{U}_{N}^{n}(\phi) T_{N}(\boldsymbol{\mu})\right] \sim \frac{1}{M_{n}} \sum_{m_{1}=0}^{M_{n}} \sum_{m_{2} \in \mathbb{Z}} N \int_{-\epsilon}^{1-\epsilon} \exp \left[2 \pi \mathrm{i}\left(\mu_{1} p(q)+\mu_{2} q\right)\right] \times \\
\times D\left(q+m_{1}, q\right) \exp \left[2 \pi \mathrm{i} N\left(S_{\phi^{n}}\left(q+m_{1}, q\right)-m_{2} q\right)\right] \mathrm{d} q .
\end{aligned}
$$


The above expression is the same as the formula that we would have obtained in determining the trace of the $n$-th propagator of an Anosov map with $D_{n}\left(q_{1}, q_{2}\right)$ replaced by $D_{n}\left(q_{1}, q_{2}\right) \exp (2 \pi \mathrm{i} \boldsymbol{\mu} \cdot \boldsymbol{z})$. Following the same steps which lead to the trace formula of Anosov maps 18, 10 we obtain

$$
\operatorname{Tr}\left[\mathrm{U}_{N}^{n}(\boldsymbol{\phi}) T_{N}(\boldsymbol{\mu})\right] \sim \sum_{\boldsymbol{z}_{\mathrm{f}}} \frac{\exp \left(2 \pi \mathrm{i} \boldsymbol{\mu} \cdot \boldsymbol{z}_{\mathrm{f}}\right)}{\sqrt{-R_{\boldsymbol{z}_{\mathrm{f}}}^{(n)}}} \exp \left[2 \pi \mathrm{i} N\left(S_{\boldsymbol{\phi}^{n}}\left(q_{\mathrm{f}}+m_{1}, q_{\mathrm{f}}\right)-m_{2} q_{\mathrm{f}}\right)\right] .
$$

Let $f_{N}$ be a truncated Fourier approximation to $f$,

$$
f_{N}(\boldsymbol{z})=\sum_{\substack{\boldsymbol{\mu} \in \mathbb{Z}^{2} \\\|\boldsymbol{\mu}\|<N^{1 / 2-\delta}}} \hat{f}_{\boldsymbol{\mu}} \exp (2 \pi \mathrm{i} \boldsymbol{\mu} \cdot \boldsymbol{z})
$$

Taking finite linear combinations, (B.13) yields

$$
\sum_{j=1}^{N}\left(\varphi_{j}, \mathrm{Op}_{N}\left(f_{N}\right) \varphi_{j}\right) \mathrm{e}^{\mathrm{i} n \omega_{j}} \sim \sum_{\boldsymbol{z}_{\mathrm{f}}} \frac{f_{N}\left(\boldsymbol{z}_{\mathrm{f}}\right)}{\sqrt{-R_{\boldsymbol{z}_{\mathrm{f}}}^{(n)}}} \exp \left[2 \pi \mathrm{i} N\left(S_{\boldsymbol{\phi}^{n}}\left(q_{\mathrm{f}}+m_{1}, q_{\mathrm{f}}\right)-m_{2} q_{\mathrm{f}}\right)\right]
$$

To conclude the proof of our claim $(\mathbb{B} .2)$, we note that, since the Fourier coefficients of $f$ are decreasing faster than any power, both

$$
\left|\sum_{j=1}^{N}\left(\varphi_{j}, \mathrm{Op}_{N}(f) \varphi_{j}\right) \mathrm{e}^{\mathrm{i} n \omega_{j}}-\left(\varphi_{j}, \mathrm{Op}_{N}\left(f_{N}\right) \varphi_{j}\right) \mathrm{e}^{\mathrm{i} n \omega_{j}}\right| \leq \sum_{j=1}^{N}\left|\left(\varphi_{j}, \mathrm{Op}_{N}\left(f-f_{N}\right) \varphi_{j}\right)\right|
$$

and

$$
\left|\sum_{\boldsymbol{z}_{\mathrm{f}}} \frac{f\left(\boldsymbol{z}_{\mathrm{f}}\right)-f_{N}\left(\boldsymbol{z}_{\mathrm{f}}\right)}{\sqrt{-R_{\boldsymbol{z}_{\mathrm{f}}}^{(n)}}} \exp \left[2 \pi \mathrm{i} N\left(S_{\boldsymbol{\phi}^{n}}\left(q_{\mathrm{f}}+m_{1}, q_{\mathrm{f}}\right)-m_{2} q_{\mathrm{f}}\right)\right]\right| \leq \sum_{\boldsymbol{z}_{\mathrm{f}}}\left|\frac{f\left(\boldsymbol{z}_{\mathrm{f}}\right)-f_{N}\left(\boldsymbol{z}_{\mathrm{f}}\right)}{\sqrt{-R_{\boldsymbol{z}_{\mathrm{f}}}^{(n)}}}\right|
$$

are rapidly decreasing, as $N$ becomes large.

\section{References}

[1] O. Bohigas, M.-J. Giannoni and C. Schmit: Characterization of chaotic quantum spectra and universality of level fluctuation laws, Phys. Rev. Lett. 52 (1984) 1-4.

[2] E. Caurier and B. Grammaticos: Extreme level repulsion for chaotic quantum Hamiltonians, Phys. Lett. A 136 (1989) 387-390.

[3] R. Scharf, B. Dietz, M. Kuś, F. Haake and M. V. Berry: Kramers' Degeneracy and Quartic Level Repulsion, Europhys. Lett. 5 (1988) 383-389.

[4] R. Scharf: Kicked rotator for a spin 1/2, J. Phys. A 22 (1989) 4223-4242. 
[5] M. Thaha, R. Blümel and U. Smilansky: Symmetry breaking and localization in the quantum chaotic regime, Phys. Rev. E 48 (1993) 1764-1781.

[6] M. I. Brin: The topology of group extensions of C-systems, Mat. Zametki/Math. Notes $18(1975 / 1976)$ 453-465/858-864.

[7] H. B. Keynes and D. Newton: The structure of ergodic measures for compact group extensions, Israel J. Math. 18 (1974) 363-389.

[8] H. B. Keynes and D. Newton: Ergodic measures for non-abelian compact group extensions, Compositio Math. 32 (1976) 53-70.

[9] M. S. M. Noorani: Ergodicity and weak-mixing of homogeneous extensions of measurepreserving transformations with applications to Markov shifts, Monatsh. Math. 123 (1997) 149-170.

[10] J. P. Keating and F. Mezzadri: Pseudo-symmetries of Anosov maps and spectral statistics, Nonlinearity 13 (2000) 747-775.

[11] P. Kurlberg and Z. Rudnick: Hecke theory and equidistribution for the quantization of linear maps of the torus, Duke Math. J. 103 (2000) 47-78.

[12] J. Bolte and S. Keppeler: Semiclassical Time Evolution and Trace Formula for Relativistic Spin-1/2 Particles, Phys. Rev. Lett. 81 (1998) 1987-1991.

[13] J. Bolte and S. Keppeler: A semiclassical approach to the Dirac equation, Ann. Phys. (NY) 274 (1999) 125-162.

[14] J. H. Hannay and A. M. Ozorio De Almeida: Periodic orbits and a correlation function for the semiclassical density of states, J. Phys. A 17 (1984) 3429-3440.

[15] M. V. Berry: Semiclassical theory of spectral rigidity, Proc. R. Soc. London Ser. A 400 (1985) 229-251.

[16] J. Bolte and S. Keppeler: Semiclassical form factor for chaotic systems with spin 1/2, J. Phys. A 32 (1999) 8863-8880.

[17] J. H. Hannay and M. V. Berry: Quantization of linear maps on a torus - Fresnel Diffraction by a periodic grating, Physica D 1 (1980) 267-290.

[18] M. Basilio de Matos and A. M. Ozorio de Almeida: Quantization of Anosov Maps, Ann. Phys. 237 (1995) 46-65.

[19] F. Haake: Quantum Signatures of Chaos, Springer-Verlag, Berlin Heidelberg, (1991).

[20] M. Berry, J. Keating and S. Prado: Orbit bifurcations and spectral statistics, J. Phys. A: Math. Gen. 31 (1998) L245-L254. 
[21] H. A. Kramers: Théorie générale de la rotation paramagnétique dans les cristaux, Proc. Acad. Amst. 33 (1930) 959-972.

[22] E. P. Wigner: Über die Operation der Zeitumkehr in der Quantenmechanik, Nachrichten der Gesellschaft der Wissenschaften zu Göttingen, Mathematisch Physikalische Klasse (1932) 546-559.

[23] J. P. Keating: The cat maps: quantum mechanics and classical motion, Nonlinearity 4 (1991) 309-341.

[24] M. C. Gutzwiller: Periodic Orbits and Classical Quantization Conditions, J. Math. Phys. 12 (1971) 343-358.

[25] M. L. Mehta: Random matrices, Academic Press Inc., Boston, MA, 2nd edn., (1991).

[26] J. P. Keating: Asymptotic properties of the periodic orbits of the cat maps, Nonlinearity 4 (1991) 277-307.

[27] W. Parry and M. Pollicott: Zeta functions and the periodic orbit structure of hyperbolic dynamics, Astérisque 187-8 (1990) 1-268.

[28] A. Gamburd, D. Jakobson and P. Sarnak: Spectra of elements in the group ring of SU(2), J. Eur. Math. Soc. (JEMS) 1 (1999) 51-85.

[29] J.-P. Serre: Linear representations of finite groups, Springer-Verlag, New York, (1977), Translated from the second French edition by Leonard L. Scott, Graduate Texts in Mathematics, Vol. 42.

[30] I. Percival and F. Vivaldi: Arithmetical properties of strongly chaotic motions, Physica D 25 (1987) 105-130.

[31] R. Aurich, F. Scheffler and F. Steiner: On the subtleties of arithmetical quantum chaos, Phys. Rev. E 51 (1995) 4173.

[32] E. B. Bogomolny, B. Georgeot, M.-J. Giannoni and C. Schmit: Arithmetical chaos, Phys. Rep. 291 (1997) 219-324.

[33] R. Aurich and J. Marklof: Trace formulae for three-dimensional hyperbolic lattices and application to a strongly chaotic tetrahedral billiard, Physica D 92 (1996) 101-129.

[34] M. Degli Esposti: Quantization of the orientation preserving automorphisms of the torus, Ann. Inst. H. Poincaré 58 (1993) 323-341.

[35] M. Degli Esposti, S. Graffi and S. Isola: Classical limit of the quantized hyperbolic toral automorphisms, Commun. Math. Phys. 167 (1995) 471-507.

[36] A. Bouzouina and S. De Bièvre: Equipartition of the eigenfunctions of quantized ergodic maps on the torus, Comm. Math. Phys. 178 (1996) 83-105. 
[37] M. Wilkinson: A semiclassical sum rule for matrix elements of classically chaotic systems, J. Phys. A 20 (1987) 2415-2423.

[38] T. Paul and A. Uribe: The Semi-Classical Trace Formula and Propagation of Wave Packets, J. Funct. Anal. 132 (1995) 192-249.

[39] J. Bolte: Semiclassical expectation values for relativistic particles with spin 1/2, to appear in: Found. Phys.; nlin.CD/0009052. 